\title{
Ca2+ signalling is critical for autoantibody-induced blistering of human epidermis in pemphigus vulgaris
}

\author{
Thomas Schmitt ${ }^{1}$, Desalegn Egu ${ }^{1}$, Elias Walter ${ }^{1}$, Anna Sigmund ${ }^{1}$, Ramona Eichkorn ${ }^{2}$, \\ Amir Yazdi ${ }^{2}$, Enno Schmidt ${ }^{3}$, Miklós Sárdy ${ }^{4}$, Ruediger Eming ${ }^{5}$, and Jens Waschke ${ }^{1}$ \\ ${ }^{1}$ Ludwig-Maximilians-Universität München Anatomische Anstalt \\ ${ }^{2}$ University Medical Center Tübingen Pharmacy \\ ${ }^{3}$ University of Lübeck \\ ${ }^{4}$ Semmelweis University \\ ${ }^{5}$ Philipps-Universität Marburg
}

September 16, 2020

\begin{abstract}
Background: Pemphigus vulgaris (PV) is a severe autoimmune skin disease. Autoantibodies in PV (PV-IgG) targeting desmoglein (DSG)1 are required for loss of desmosome adhesion and skin blistering. Objectives: The aim was to connect the single steps of a Ca2+ flux dependent pathway and show it's importance for the pathogenesis of pemphigus vulgaris. Methods: Applied methods include immunoprecipitation, Ca2+-flux analysis with FURA2-AM, analysis of protein phosphorylation with Western-blotting, Immunofluorescence, investigation of loss of adhesion in dissociation assays and human skin ex-vivo blistering model. Results: PV-IgG but not a DSG3-specific monoclonal antibody (AK23) caused Ca2+ influx in primary human keratinocytes. Phosphatidyl inositol 4 kinase- $\alpha$ (PI4K) interacts with DSG1 but not DSG3. Its downstream targets, phospholipase-C- $\gamma 1$ (PLC) and protein-kinase-C $\alpha$, similar to p38MAPK interacted with both. PLC was activated by PV-IgG but not by AK23. PLC regulates the release of Inositol-1,4,5-trisphosphate (IP3) causing IP3-receptor (IP3R) activation and $\mathrm{Ca} 2+$ flux from the endoplasmic reticulum into the cytosol. This stimulates Ca2+-release-activated-channels (CRAC) causing Ca2+ influx. Inhibitors against PI4K, PLC, IP3R or CRAC effectively blocked PV-IgG-induced Ca2+ influx and ameliorated alterations of DSG1 and 3 localization, keratin filament retraction, actin disruption and loss of cell adhesion in vitro. Moreover, inhibiting PLC was protective against blister formation, redistribution of DSG1 and 3 and actin disruption in human skin ex vivo. Conclusions: These results demonstrate that Ca2+-mediated signalling is important for pemphigus pathology and dependent on the autoantibody profile, with different roles for the Dsg1 and Dsg3 complexes. PLC could be an interesting pharmaceutical target.
\end{abstract}

\section{INTRODUCTION}

The skin as the main outer barrier against pathogens, chemicals and other hazards is an important organ. The autoimmune disease pemphigus vulgaris (PV) affects the mucosa and epidermis . Disruption of the skin barrier, caused by often extensive blisters, can lead to severe complications and high mortality if untreated . Current first-line treatments are systemic high-dose long term corticosteroids with or without further immunosuppressants or rituximab combined with rapidly tapered corticosteroids, both associated with high rates of severe adverse events. Other treatment options such as intravenous-immunoglobulin G (IV-IgG) or immuno-adsorption also have drawbacks, including availability of donor IgG, immunosuppressive effects and short-term effectiveness . Understanding the mechanisms of PV pathology and finding specific treatment options are thus the main goals of current research.

$\mathrm{PV}$ is caused by autoantibodies (PV-IgG) including IgG against the desmosomal proteins desmoglein (DSG 
)1 and 3 and autoantibodies against other target proteins. Desmosomes are adhesive cell-cell contacts in the epidermis, in which $D S G$ s are connected to plaque proteins including desmoplakin $(D P)$ which links them to the keratin cytoskeleton .

Desmosomes provide mechanical stability, but also partake in cellular signalling. In pemphigus, loss of desmosomal adhesion is caused by both direct inhibition of $D S G$ binding and cellular signalling mechanisms

It is well-known that the clinical phenotype of PV correlates with the autoantibody profile and anti- $D S G$ 1-IgG are believed to be required for epidermal blistering. It is thus possible that anti-DSG 1-IgGs modulate specific signalling pathways involved in loss of desmosomal adhesion . Indeed, $\mathrm{Ca}^{2+}$ influx in human keratinocytes was shown to be induced by PV-IgG including autoantibodies against $D S G 1$ but not by AK23, a $D S G$ 3-specific IgG . PV-IgG is known to lead to activation of phospholipase-C $\gamma 1$ (PLC) generating inositol-1,4,5-trisphosphat (IP3) and increasing the intracellular $\mathrm{Ca}^{2+}$ concentration . In line with this, an interaction between $D S G 1$ and PI4K (phosphoinositole-4-kinase $\alpha$ ), upstream of $P L C$, was predicted .

Further downstream, IP3 activates IP3R(Inositol-trisphosphate-receptor), releasing $\mathrm{Ca}^{2+}$ from the endoplasmic reticulum (ER) into the cytosol . $\mathrm{Ca}^{2+}$ flux from the ER into the cytosol stimulates $\mathrm{Ca}^{2+}$-releaseactivated-channels (CRAC) causing further $\mathrm{Ca}^{2+}$ influx. $\mathrm{Ca}^{2+}$ activates protein kinase-C $\alpha(P K C)$, which was shown to also be important for PV-IgG-induced loss of keratinocyte adhesion and skin blistering by mechanisms including depletion of $D S G 3$, keratin retraction and loss of cell adhesion in vitro and in mice in vivo .

\section{RESULTS}

$P V$-IgG induces DSG1-dependent $\mathrm{Ca}^{2+}$ flux via IP3R and CRAC in vitro

$I P 3 R$ is required to redistribute $\mathrm{Ca}^{2+}$ from the ER to the cytosol but not sufficient to increase intracellular $\mathrm{Ca}^{2+}$ concentrations. To achieve this, STIM1 and ORAI1 form the so-called CRAC $\left(\mathrm{Ca}^{2+}\right.$-release-activatedchannel), in whichSTIM1 serves as a $\mathrm{Ca}^{2+}$ concentration sensor at the ER membrane. Upon low $\mathrm{Ca}^{2+}$, STIM1 contactsORAI1 at the plasma membrane to replenish the ERs $\mathrm{Ca}^{2+}$ store. We found IP3R and ORAI1 to be equally distributed in all epidermal layers, whereas STIM1 immunostaining was more prominent in the granular layer (Figure 1a). Similar to previous studies, PV-IgG containing autoantibodies against $D S G$ 1 and DSG 3 (Table 1) but not IgG from healthy volunteers led to an increase in intracellular $\mathrm{Ca}^{2+}$ in NHEK cells (Figure 1b/S1a). In agreement with a previous report, the $\mathrm{Ca}^{2+}$ flux was anti- $D S G$ 1-dependent, as no influx was observed for the anti-DSG 3-mAb AK23 (Figure 1b/S1a). Inhibition of CRAC with BTP-2, $I P 3 R$ with Xestospongin $\mathrm{C}$ (Xest), $P L C$ with U-73122 or PI4K with GSK-F1 abolished the $\mathrm{Ca}^{2+}$ flux (Figure 1b/S1a). Co-immunoprecipitation demonstrated that PI4K interacts with $D S G 1$ but not with $D S G 3, D S G 1$ s downstream target $P L C$ as well asp38MAPK (Figure 1c+d/S1b), which is known to be strongly involved in PV pathogenesis, are part of both complexes. So far, only the interaction of $p 38 M A P K$ with $D S G 3$ was shown . Phosphorylated $P K C$, reported to be responsible for the cytoskeleton reorganization and DSG 3 depletion, was found in both complexes (Figure 1c+d). PV-IgG but not AK23 induced phosphorylation of $P L C$ (Figure 1e+f). Taken together, these results demonstrate that a $D S G$ 1-specific complex exists which, induced by PV-IgG, can initiate a $\mathrm{Ca}^{2+}$ flux dependent pathway.

Inhibition of $\mathrm{Ca}^{2+}$ flux is protective against PV-IgG-induced pathogenic effects in vitro

PV-IgG caused loss of keratinocyte adhesion in dispase-based dissociation assays. Treatment with inhibitors against PI4K, PLC , IP3R or CRAC added $1 \mathrm{~h}$ before PV-IgG effectively blocked cell monolayer fragmentation after $2 \mathrm{~h}$ and $24 \mathrm{~h}$. After $24 \mathrm{~h}$, inhibition of $P L C$ was the most effective and even reduced the effect of AK23 (Figure 2a-d/S2a-d).

The role of $\mathrm{Ca}^{2+}$ signalling for PV-IgG-mediated effects on $D S G 1$ and $D S G 3$ localization, keratin retraction and reorganization of cortical actin was assessed by immunostaining and F-actin staining. After $24 \mathrm{~h}$ of PVIgG treatment, $D S G 1$ and $D S G 3$ immunostaining was fragmented at cell borders and partially relocated 
to the cytosol. Treatment with inhibitors for PI $4 K, P L C, I P 3 R$ or CRAC, ameliorated pathogenic effects (Figure 3a/S3a-c).

Retraction of keratin filaments from the cell borders was visible after $24 \mathrm{~h}$ of PV-IgG treatment. Treatment with the inhibitors ameliorated the effect (Figure 3b/S3d). Similarly, cortical F-actin also showed defects especially close to intercellular gaps after PV-IgG treatment, which was ameliorated by the respective inhibitors (Figure 3/3S). F-actin staining was more pronounced following inhibition of $P L C$ compared to controls (Figure 3/S3) indicating that PLC basal activity might regulate actin remodelling.

Inhibition of $\mathrm{Ca}^{2+}$ flux is protective against PV-IgG-induced pathogenic effects in human skin ex vivo

In human skin samples PV-IgG induced blistering (Figure 4a) where typical tombstoning was observed (Figure 4/S4). At the blistering sites, PV-IgG was enriched and in non-affected regions it was deposited at cell borders (Figure S4a). DSG 1 and 3 staining was fragmented and redistributed to the cytosol in response to incubation with autoantibodies (Figure 4c/S4b). Moreover, actin staining was almost abolished at blistering sites (Figure 4d). All pathogenic effects were completely blocked by inhibition of $P L C$ (Figure 4/S4). This demonstrates that PLC is important for PV-IgG-induced blistering of human skin.

\section{DISCUSSION AND CONCLUSION}

The roles of $\mathrm{Ca}^{2+-}$ influx and PLC for pemphigus vulgaris pathogenesis

$\mathrm{Ca}^{2+}$ flux-dependent signalling plays an important role in pemphigus pathogenesis. Inhibiting $\mathrm{Ca}^{2+}$ signalling blocks PV-IgG-induced loss of keratinocyte adhesion in vitro and blistering in human skin ex vivo (Figure 5). Binding of PV-IgG toDSG 1 [1], associated with PI $4 K$ and $P L C$, activates $P L C$ and releases IP3 [2]. IP3 activates IP3Rthereby releasing $\mathrm{Ca}^{2+}$ from the ER into the cytosol [3]. Low ER $\mathrm{Ca}^{2+}$ concentration activates STIM1 at the ER membrane. STIM1 contacts ORAI1 by forming the CRAC and thereby causing $\mathrm{Ca}^{2+}$ influx from the extracellular space [4]. With the IP3R still active, this further increases the $\mathrm{Ca}^{2+}$ concentration in the cytosol. Cytosolic $\mathrm{Ca}^{2+}$ finally activates $P K C[5]$, which destabilizes the desmosome by keratin retraction and depletion of $D S G 1$ and $D S G 3$ [6].

The complete mechanism of action of $P K C$ is still unknown. However, it presumably affects desmosome turnover on several levels. Upon PV-IgG treatment $P K C$ has been proposed to translocate to the desmosomal plaque. Cytoskeletal components such as keratin 8 and 18 are substrates of $P K C$ possibly inducing keratin filament retraction. Similarly, $P K C$ affects the actin cytoskeleton, mostly causing disassembly and reduced anchorage and may also modulate desmosome turn-over by modulating the actin-binding adducin . Moreover, $P K C$ phosphorylates $D P$ which is involved in destabilizing the desmosome, and caused $D S G 3$ fragmentation and acantholysis in pemphigus passive-immune-transfer mouse models which supports the observations that inhibiting $P K C$ ameliorated PV-IgG-induced loss of adhesion in vitro .

$\mathrm{Ca}^{2+}$ has very profound effects on desmosome regulation. The stability of newly formed desmosomes is dependent on extracellular $\mathrm{Ca}^{2+}$. After several days, desmosomes become hyper-adhesive and independent of extracellular $\mathrm{Ca}^{2+}$. Hyper-adhesive desmosomes are also less sensitive to PV-IgG . Inhibiting PKC causes a rapid transformation from $\mathrm{Ca}^{2+}$-dependent to $\mathrm{Ca}^{2+}$-independent desmosomes . Active $P K C$ is associated with $D S G 1$, which is known to be important for regulation of epidermal differentiation . It is thus conceivable that $D S G 1$ controls desmosome adhesion and skin differentiation at least in part by controlling $\mathrm{Ca}^{2+}$ levels in keratinocytes.

Loss of cell adhesion in response to AK23, which did not cause $\mathrm{Ca}^{2+}$ influx and disrupts DSG 3 but notDSG 1 was ameliorated significantly by inhibiting $P L C$. This indicates that $P K C$, which was observed to be involved in DSG 3 depletion in vivo and in vitro may be activated independently of $\mathrm{Ca}^{2+}$ or baseline activity is sufficient. It was reported, that PV-IgG against thyroid peroxidase and other targets might also be able to activate $\mathrm{Ca}^{2+}$ flux possibly via $P L C$. This indicates that these non-desmoglein antibodies might play a crucial role for disease severity and relapse.

It is unlikely that $P K C$ is the sole downstream pathway activated by antibodies against $D S G 1$. In human 
epidermis, inhibition of $P K C$ alone under conditions which were effective in vitroand in mice in vivo, was not enough to block blistering in human skin ex vivo. In contrast, inhibition of $p 38 M A P K$, was protective in murine skin in vivo and human epidermis but not in mucosa ex vivo. Since we observed that $p 38 M A P K$ is associated with both $D S G 1$ and $D S G 3$, these data are compatible with the hypothesis that antibodies against $D S G 1$ and $D S G 3$ or other targets are required for skin blistering. $P L C$ might also modulate other events regulated by $p 38 M A P K$ such as actin remodelling via $R H O A$.

This demonstrates that PV pathology is dependent on the antibody profiles, different anti-DSG1/DSG 3 ratios as well as different target epitopes might play an important role. This might influence the clinical phenotype by inducing specific signalling responses in keratinocytes. More studies are required to delineate the functional interplay between the complex mechanisms involved in pemphigus pathogenesis. Nevertheless, the data presented here can explain for the first time why autoantibodies against $D S G 1$ but not $D S G 3$ are required for epidermal involvement in PV. SincePI4K upstream of $\mathrm{Ca}^{2+}$ signalling specifically interacts with $D S G 1$ but not with $D S G 3$, this knowledge may allow a new strategy to develop $D S G 1$-specific treatment options in pemphigus.

\section{MATERIALS \& METHODS}

\section{Cell Culture}

For all in vitro experiments, primary normal human epithelial keratinocytes (NHEK) in passage 3-6 where used. NHEKs were generated at the Universitäts-Hautklinik Tübingen, approved by the medical ethical committee of the Eberhard Karls University Tübingen (ethical approval: 547/2011BO2). The cells were isolated from juvenile foreskin derived from patients, who have given written informed consent. The epidermis was isolated using $50 \mathrm{mg} / \mathrm{ml}$ dispase II (Roche, Switzerland). Cells were separated using a trypsin-EDTA solution (Merck, Germany). The cells were cultivated in a humidified, $5 \% \mathrm{CO}_{2}$ atmosphere at $37{ }^{\circ} \mathrm{C}$ in CnT-07 medium (CELLnTEC, Switzerland) containing $10 \mu \mathrm{g} / \mathrm{ml}$ gentamycin and $0.25 \mu \mathrm{g} / \mathrm{ml}$ amphotericin B. The cells were kept under low calcium conditions $\left(0.06 \mathrm{mM} \mathrm{Ca}^{2+}\right) .24 \mathrm{~h}$ prior to treatment, the confluent cells were differentiated by adding $1.8 \mathrm{mM} \mathrm{Ca}^{2+}$. For experiments the cells were incubated with vehicle or mediators 1:50 in DMSO with final concentrations: CRAC inhibitor BTP-2 (Merk, Germany), $10 \mu \mathrm{M}$; PI4K inhibitor GSK-F1 (SYNkinase, Australia), $10 \mathrm{nM}$; PLC inhibitor U-73122 (Santa Cruz, USA), $4 \mu \mathrm{M}$; or IP3R inhibitor Xestospongin C (Abcam, USA), $2 \mu \mathrm{M}$ for $1 \mathrm{~h}$ before IgG treatment.

\section{Patient IgGs}

\section{[Table1]}

\section{Immunostaining}

Cells were grown on glass coverslips and fixed with $2 \%$ paraformaldehyde for 5 min (for rabbit-anti-DSG 3-mAb (Biozol, Germany), rabbit-anti- $D S G$ 1-pAb (Abclonal, USA), mouse-anti- $D S G$ 1-mAb (Progen, Germany) mouse-anti-GAPDH-mAb (Santa Cruz, USA), mouse-anti-HSP60-mAb (Thermo Scientific, USA) rabbit-ant-IP3R -pAb (Abcam, USA), mouse-anti-Orai1-mAb (Santa Cruz, USA), rabbit-anti-PI4K a-mAb (Abbexa, United Kingdom), rabbit-Anti-p- $P K C \alpha$-mAb (Abcam, USA), rabbit-anti-p- $P L C \gamma 1-\mathrm{mAb}$ (Cell signaling, USA), rabbit-anti-Stim1-mAb (Cell signaling, USA) and in ethanol $\left(-20{ }^{\circ} \mathrm{C}\right)$ shaking on ice for 30 min and acetone $\left(-20^{\circ} \mathrm{C}\right.$ ) for 3 min (For mouse-anti-cytokeratin-pan-mAb (Sigma Aldich, USA) 1:100-200. Paraformaldehyde fixed cells were permeabilized with $1 \%$ Triton X-100 in PBS for 5 min (for acetone fixed cells no permeabilization was necessary), the cells were blocked with $3 \%$ bovine serum albumin (BSA) and $1 \%$ normal goat serum in PBS for 30 min. Primary antibodies were applied overnight at $4{ }^{\circ} \mathrm{C}$. Cy3 coupled goat-anti-rabbit/mouse/human secondary antibodies (Dianova, Germany) and Alexa-488-phalloidin (Life technologies, USA) were incubating for $1 \mathrm{~h}$ and DAPI 1:10.000 for $15 \mathrm{~min}$. The cover slips were mounted with $2 \%$ n-propyl-gallate and evaluated with a SP5.II confocal microscope with a 63x NA 1.4 PL APO objective (Leica, Germany). After heating to $60{ }^{\circ} \mathrm{C}$ for $30 \mathrm{~min}$ the skin slices were treated the same, except for $1 \mathrm{~h}$ permeabilization. The cells were washed $3 \mathrm{x}$ with PBS in between each step except ethanol to acetone.

Ratiometric intracellular $\mathrm{Ca}^{2+}$ measurements 
Fura-2AM (Thermo Fisher, USA) was used to measure intracellular $\mathrm{Ca}^{2+}$ in real time. The cells were grown in an 8-well $\mu$-slide (Ibidi, Germany). Mediators were contained in all incubation steps and during the measurement. A mix of $1 \mu \mathrm{M}$ Fura-2AM and $0.02 \%$ Pluronic (Thermo Fisher, USA) was applied for $20 \mathrm{~min}$ in measurement buffer $\left(140 \mathrm{mM} \mathrm{NaCl}, 3.6 \mathrm{mM} \mathrm{KCl}, 2.6 \mathrm{mM} \mathrm{CaCl} 2\left(\mathrm{H}_{2} \mathrm{O}\right)_{2}, 0.5 \mathrm{mM} \mathrm{MgSO}_{4}\right.$, $0.5 \mathrm{mM} \mathrm{NaH} \mathrm{PO}_{4}\left(\mathrm{H}_{2} \mathrm{O}\right)_{2}, 2 \mathrm{mM} \mathrm{NaHCO} 3,5 \mathrm{mM}$ HEPES and $5 \mathrm{mM} \mathrm{D}+$ Glucose, $\left.\mathrm{pH} 7.35\right)$ at $37{ }^{\circ} \mathrm{C}$. The cells were washed twice with measurement buffer. Measuerments were performed using MetaFluor (Moleculardevices, USA) on an Axio Observer A1 (Zeiss, Germany) with a Polychrome V (Till Photonics, Germany), a CoolSNAP-Hq2 digital camera (Photometrics, USA) and a Fura-2 filter set.

\section{Co-Immunoprecipitation}

NHEK were cultured in T75-Cell flasks, washed $1 \mathrm{x}$ with PBS $+10 \mathrm{~g} / \mathrm{l}$ EDTA and $2 \mathrm{x}$ with PBS. $1 \mathrm{ml} \mathrm{Ca}{ }^{2+}$ free-PBS with $\frac{1}{2} \mathrm{NaCl}$ concentration $+1 \%$ triton-X100 $+1 \%$ Nonoxinol- $40+0.1 \%$ sodium-dodecyl-sulfate + cOmplete (Merk, USA) was used for cell lysis shaking 15 min on ice. Buffer without SDS, $0.5 \%$ TritonX100 and $0.1 \%$ Nonoxinol-40 was used for washing. The cells were mechanically detached and sheared with a $5 \mathrm{ml}$ syringe and 10G needle (B.Braun, Germany) 10x. The resulting suspension was centrifuged at 4 $\operatorname{deg} \mathrm{C}$ for $15 \mathrm{~min}$ at $13.000 \mathrm{rpm}$ and the pellet was removed. The protein amount was determined with a commercial Pierce BCA protein assay kit. The supernatant was added to buffer-washed Agarose G beads (Milipore, USA) adding about $600-1000 \mu \mathrm{g} /$ protein. After $1.5 \mathrm{~h}$ on a rotator, the beads were removed via centrifuging for 2 min at $4{ }^{\circ} \mathrm{C}, 8.000 \mathrm{rpm}$ and the supernatant was mixed with $1.5 \mu \mathrm{l}$ of antibody or normal rabbit IgG, $1 \mathrm{mM} \mathrm{Ca}^{2+}$ and $0.5 \mathrm{mM} \mathrm{Mg}{ }^{2+}$ and rotated for $3 \mathrm{~h}$. The mixture was added to washed beads and rotated at $4{ }^{\circ} \mathrm{C}$ over night. The beads were washed $3 \mathrm{x}\left(1 \mathrm{~min} 4{ }^{\circ} \mathrm{C}, 3.000 \mathrm{rpm}\right)$. Proteins were released from the beads using $27 \mu \mathrm{l} 95{ }^{\circ} \mathrm{C}$, 3x Lämmli-buffer and beads were removed by centrifuging $\left(5 \min 4{ }^{\circ} \mathrm{C}\right.$, $8.000 \mathrm{rpm})$.

\section{Cell lysis, gel electrophoresis and Western blotting}

Cells were cultured in 24-well-plates. Lysates were fractioned into a soluble cytosolic and insoluble cytoskeletal bound fraction using triton-extraction-buffer (0.5\% Triton X-100, 50 mmol/l MES, 25 mmol/l EGTA, $5 \mathrm{mmol} / \mathrm{l} \mathrm{MgCl} 2, \mathrm{pH}$ 6.8, $0.1 \%$ pepstatin+aprotinin+leupeptin, $1 \% \mathrm{PMSF}$ ) for $10 \mathrm{~min}$ on ice under gentle shaking. The pellet $=$ cytoskeletal fraction was separated at $14.000 \mathrm{rpm}$ for $10 \mathrm{~min}$ at $4^{\circ} \mathrm{C}$ and the supernatant=cytosolic fraction was retrieved. The pellet was washed $1 \mathrm{x}$ and lysed with ultrasound in SDS lysis buffer (25 mM HEPES, $2 \mathrm{mM}$ EDTA, $25 \mathrm{mM} \mathrm{NaF}, 1$ \% SDS, pH 7.6, cOmplete (Merk, USA)). Protein amount was determined with a commercial Pierce BCA protein assay kit. Western-blotting was performed, using a standard wet blotting protocol on nitrocellulose membranes (Life Technologies, USA). Membranes were blocked with ROTI(r)Block (Carl Roth, Germany) 1:10 in Tris-buffered saline with $0.05 \%$ tween (TBST) for $1 \mathrm{~h}$ antibodies were used overnight at $4 \mathrm{degC}$ in $5 \%$ BSA in TBST 1:1000, except anti-p-PKC $\alpha$ (1:20.000). Anti-rabbit/mouse horseradish-peroxidase-coupled secondary antibodies (Dianova, Germany) were used 1:10.000 in TBST for $1 \mathrm{~h}$ and visualized with self-made ECL solution on a FluorchemE developer (Protein Simple, USA).

Dispase-based dissociation assay

After incubation, confluent cell monolayers were washed with Hank's buffered saline solution (HBSS) and subjected to $2.4 \mathrm{U} / \mathrm{ml}$ dispase II (Sigma Aldrich, USA) in HBSS for 20 min at $37^{\circ} \mathrm{C}, 95 \%$ humidity and 5 $\% \mathrm{CO}_{2}$. After detachment of the monolayer the reaction was stopped by adding $200 \mu \mathrm{l}$ HBSS. Defined shear stress was applied with an electrical pipette. Images for fragment counting were taken using a binocular microscope (Leica, Germany) and an EOS 600D camera (Canon, Japan).

\section{Human skin samples}

Skin biopsies were taken from body donors received by the Institute of Anatomy and Cell Biology, LudwigMaximilian-Universität München, Germany. Body donors had given written informed consent for use of tissue samples for research purposes. The procedure was approved by the Ethics Committee of the Faculty of Medicine (project no. 249-12). Only samples from body donors deceased for $<24 \mathrm{~h}$ were used. Each 
epidermis piece $\sim 4 \mathrm{~cm}^{2}$ was excised from the shoulder region and divided into $\sim 1 \mathrm{~cm}^{2}$ pieces.

Either $50 \mu \mathrm{l}$ of the PLC -inhibitor U-73122, $4 \mu \mathrm{M}$ in DMSO/PBS 1:50 or vehicle were injected intraepidermally, followed by floating incubation in $5 \mathrm{ml}$ Dulbecco's modified Eagle's medium, containing 10 $\%$ FCS, $50 \mathrm{U} / \mathrm{ml}$ penicillin and $50 \mathrm{mg} / \mathrm{ml}$ streptomycin, with the epidermis facing upwards at the liquidair-interface without additional support at $95 \%$ humidity, $5 \% \mathrm{CO}_{2}$ and $37{ }^{\circ} \mathrm{C}$. After $1 \mathrm{~h}$ incubation, either PV2 or IgG obtained from healthy volunteers, purified for experimental use by protein A affinity chromatography as described previously was injected. Injections were performed using a 0.01-1 ml syringe (B.Braun, Germany), and a 0.3x12 mm BL/LB 30Gx1/2" needle (B.Braun, Germany). After $24 \mathrm{~h}$, shear stress was applied using a rubber piece. The skin pieces were cut and processed for either hematoxylin and eosin $(\mathrm{H} \& \mathrm{E})$ or immunostaining.

\section{HEE-staining}

Samples were embedded in tissue freezing medium (Leica Biosystems, Germany) and $7 \mu \mathrm{m}$ sections were made using a CryoStar NX70 Kryostat (Thermo Fisher, USA). The resulting sections were stained with H\&E according to standard protocols. For morphometric analysis, images were captured at $200 \times$ magnifications using a light DMI8-Microscope (Leica, Germany).

\section{Statistical analysis}

Data were analysed in Excel (Microsoft, USA) and compared using one/two-way-ANOVA followed by Bonferroni-post-hoc-test (for Gaussian-distributed samples) using Graphpad Prism (Graphpad Software, USA). Error bars represent SEM. Significance was assumed with p [?] 0.05. Data are shown as mean +SEM. Each n represents one independent experiment.

\section{Author contributions}

Thomas Schmitt: Designing and conducting experiments, acquiring and analyzing data, writing the manuscript

Desalegn Egu: Conducting experiments

Elias Walter: Designing experiments, conducting preliminary experiments

Anna Sigmund: Contributing to experiment conduction

Ramona Eichkorn: Providing primary cells

Amir Yazdi: Providing primary cells

Enno Schmidt: Providing patient sera

Miklos Sardy: Providing patient sera

Rudiger Eming: Providing patient sera

Jens Waschke: Desingning experiments, analyzing data, writing the manuscript

\section{Data Availability}

The raw data supporting the conclusions of this manuscript will be made available by the authors, without undue reservation, to any qualified researcher.

\section{Acknowledgements}

We thank Silke Gotschy, Martina Hitzenbichler, and Sabine Muhlsimer for their excellent technical assistance as well as Jessica Plewa, Michael Becker, and Axel Unverzagt for assistance with human body donors. All PV samples were received from patients who had given written informed consent. A positive ethics vote was given for: PV1, University clinic Lubeck (Az 12-178), PV2 University Clinic Budapest (249-12), PV3 University Clinic Giessen/Marburg (Studie 20/14). All patients suffered from PV with mucocutaneous involvement. 
The study was supported by DFG FOR 2497 to JW and AY and in part by structural funds of the Excellence Cluster Precision Medicine in Chronic Inflammation (EXC 2167) from the Deutsche Forschungsgemeinschaft to ES.

References

Table 1: PV-IgG used in this study

\begin{tabular}{lll}
\hline IgG name & ELISA score for anti- $D S G 1$-IgG, U/ml & ELISA score for anti-DSG3-IgG, U/ml \\
\hline PV1 & 1207.00 & 3906.00 \\
PV2 & 57.60 & 177.00 \\
PV3 & 981.00 & 698.00 \\
\hline
\end{tabular}

Figure 1: a) Immunostaining of $\mathrm{Ca}^{2+}$ channel proteins in human skin sections ( $\mathrm{n}=3$, scale bar $\left.25 \mu \mathrm{m}\right)$. b) $\mathrm{Ca}^{2+}$ flux measurements upon IgG addition performed using FURA-2-AM in NHEK cells. Each curve represents 1 independent experiment, showing an average of 8 individual randomly selected measured cells, occasional non-responding cells were not included $(n=4)$. c) Co-immunoprecipitation experiments using NHEK cells, precipitating with anti- $D S G$ 1-IgG (n[?]3). d) Co-immunoprecipitation experiments using NHEK cells, precipitating with anti- $D S G$ 3-IgG (n[?]3). e) Representative Western blot showing the phosphorylation of $P L C$ after $2 \mathrm{~h} \mathrm{IgG}$ treatment. f) Quantification of $P L C$ phosphorylation in Western blot after $2 \mathrm{~h} \mathrm{IgG}$ treatment $(\mathrm{n}=6)$.

Figure 3: a) Immunostaining of $D S G 1$ (rabbit-pAb, Abclonal, USA) in NHEK cells. c) Immunostaining of keratin filaments in NHEK cells. White arrows: PV-IgG-induced fragmentation of DSG 1 staining or keratin and actin reorganization after incubation for $24 \mathrm{~h}$; red arrows: strengthening of the cortical actin (n[?]3, scale bar $25 \mu \mathrm{m})$.

Figure 4: a) Quantitative evaluation of blister formation in human skin slices ex vivo. b) Representative microscopic images of HE staining of human skin slices after IgG treatment for $24 \mathrm{~h}(\mathrm{n}=3)$. c) Immunostaining of DSG 1 (mouse-mAb, Progen, Germany) in human skin slices after PV2 treatment. d) F-actin staining in human skin slices after PV2 treatment. Each set of images shows a 4x zoom of the blister roof and bottom. Green arrows: Missing staining at the cell border; White arrows: Remaining basal cells (tombstoning) with reduced staining $(\mathrm{n}=3$, scale bar $=50 \mu \mathrm{m})$.

Figure 5 : Schematic of the $\mathrm{Ca}^{2+}$ flux-dependent signalling pathway in keratinocytes upon PV-IgG binding. 

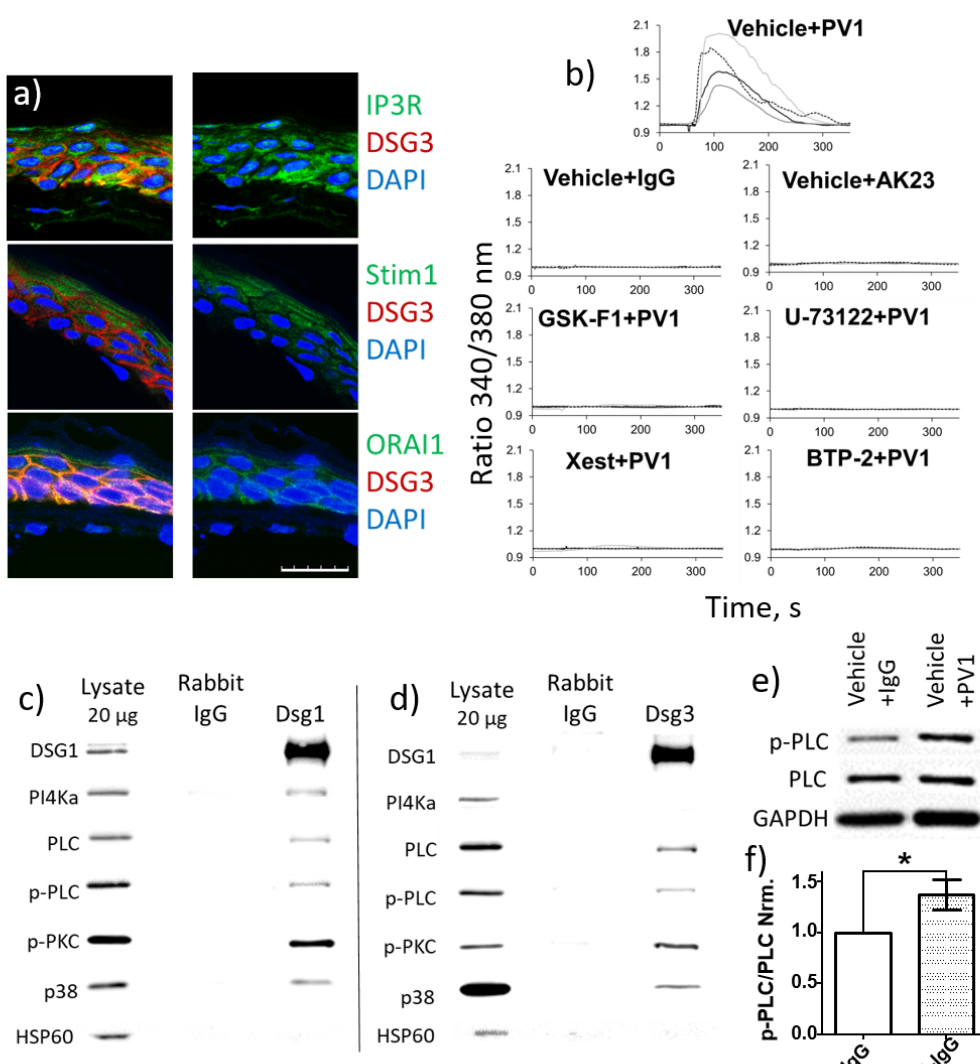

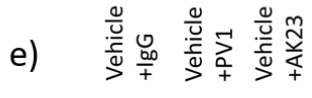

p-PLC - - -

$\mathrm{PLC}-\longrightarrow$ $\mathrm{GAPDH}=$

f)

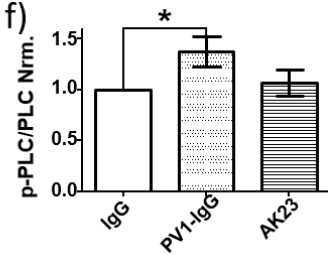




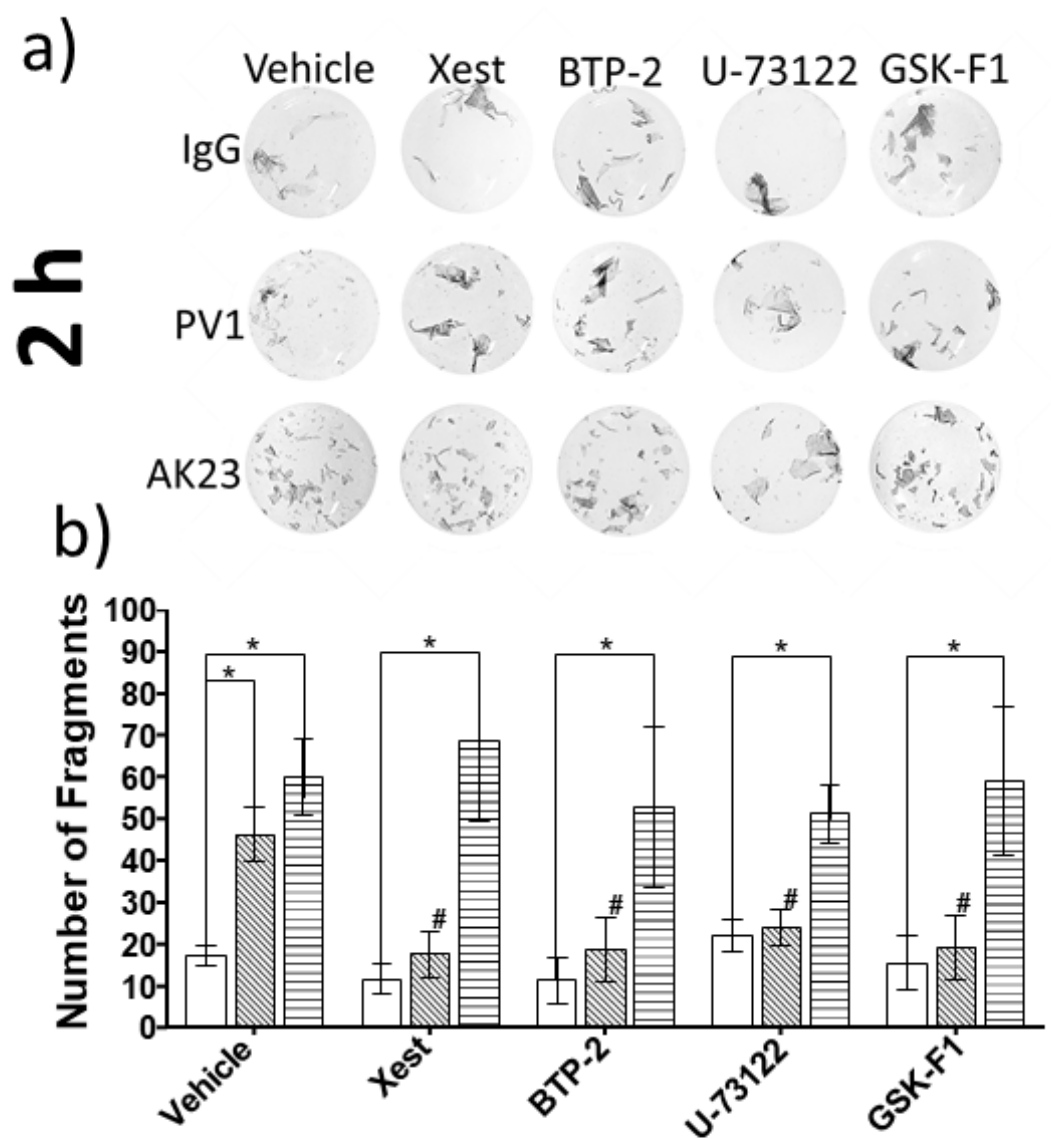

C) Vehicle Xest BTP-2 U-73122 GSK-F1 $\operatorname{lgG}$
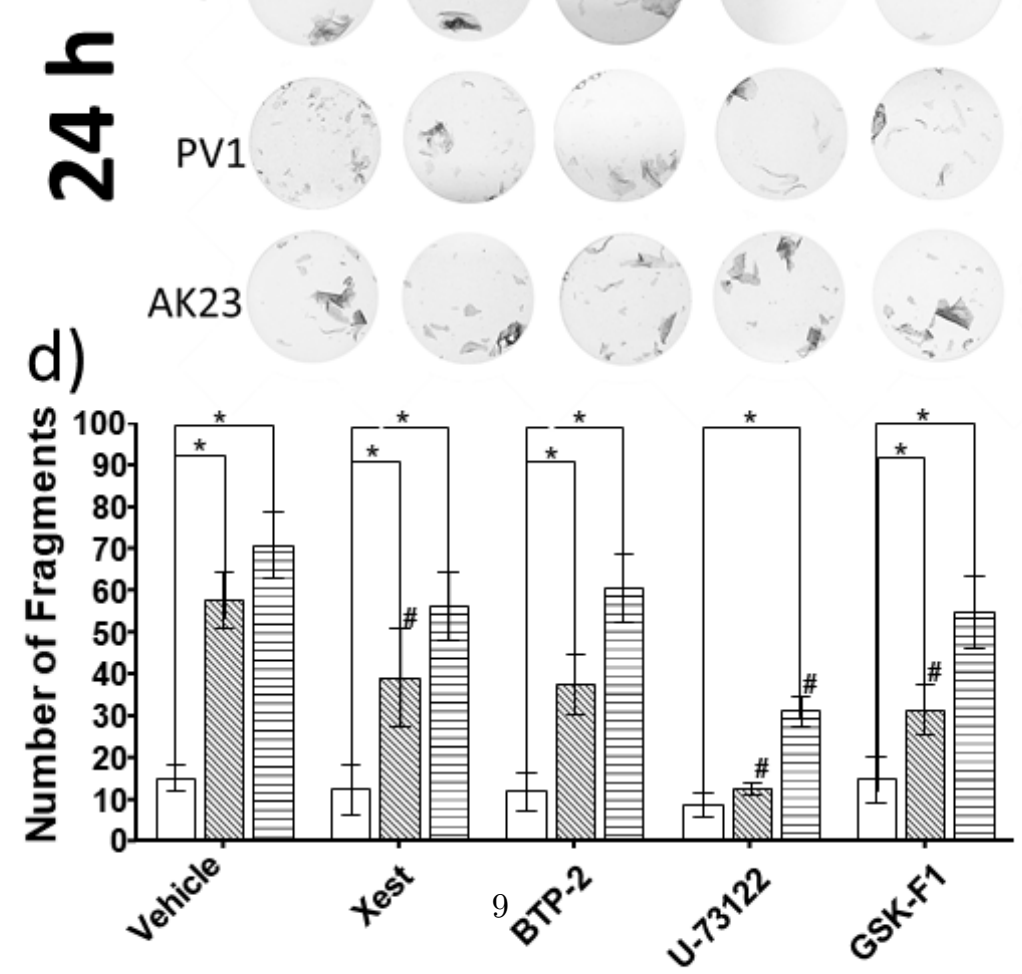

$\square$ IgG $\mathbb{P}$ P1 $\boxminus A K 23$ \#Significant to vehicle 


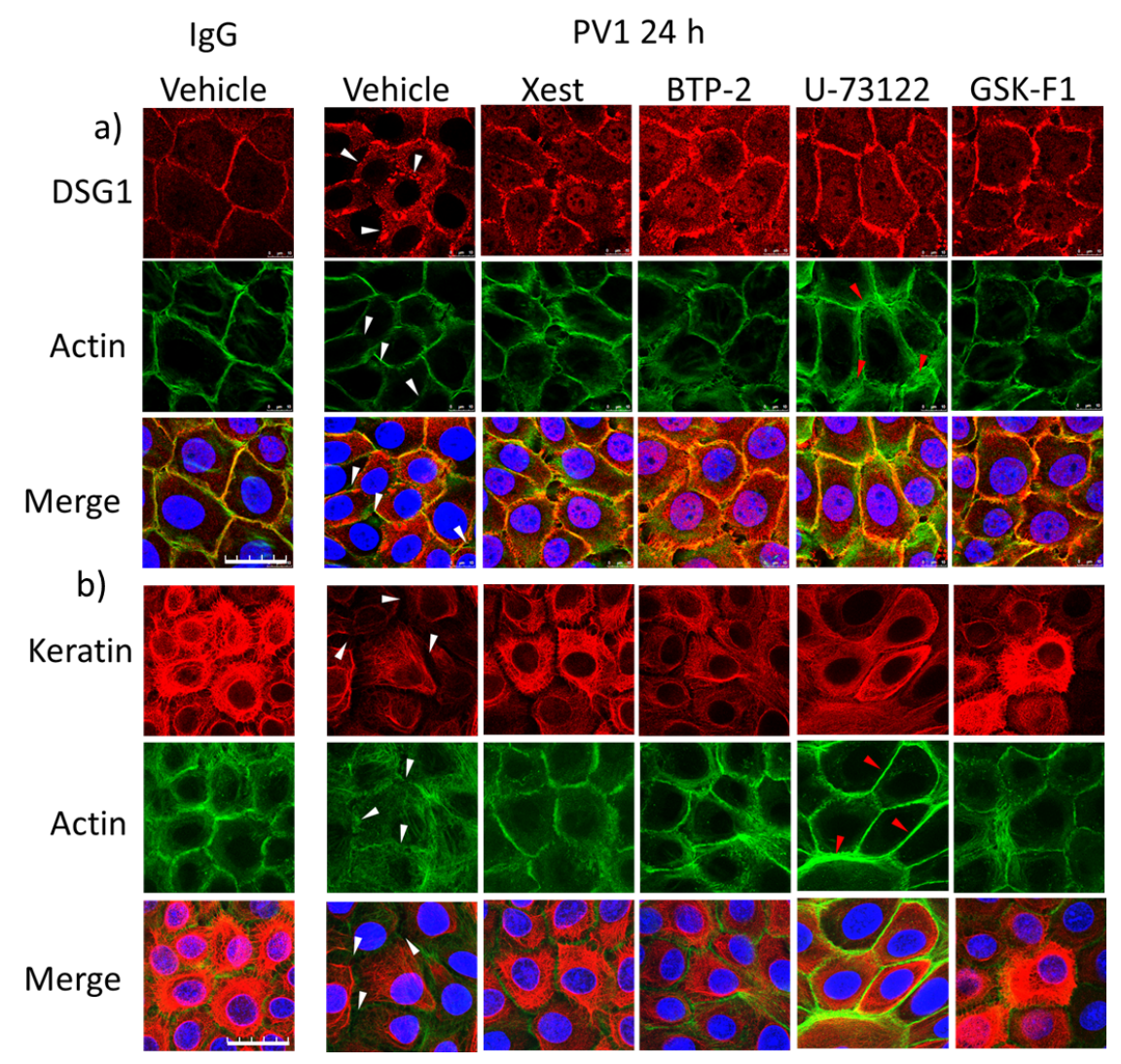




\begin{tabular}{|c|c|c|c|c|c|}
\hline \multirow[b]{2}{*}{ a) } & \multicolumn{5}{|c|}{ IgG } \\
\hline & $\begin{array}{l}\text { Max rel. } \\
\text { cleft legth }\end{array}$ & $\begin{array}{l}\text { Tot. rel. cleft } \\
\text { length }\end{array}$ & $\begin{array}{l}\text { Max cleft } \\
\text { length }\end{array}$ & total cleft length & $\begin{array}{c}\text { Nr. of } \\
\text { Blisters }\end{array}$ \\
\hline Vehicle & 0 & 0 & $0 \mu \mathrm{m}$ & $0 \pm 0 \mu \mathrm{M}$ & none \\
\hline \multirow[t]{3}{*}{ U-73122 } & 0 & 0 & $0 \mu \mathrm{m}$ & $0 \pm 0 \mu \mathrm{M}$ & none \\
\hline & \multicolumn{5}{|c|}{ PV2 } \\
\hline & $\begin{array}{l}\text { Max rel. } \\
\text { cleft legth }\end{array}$ & $\begin{array}{c}\text { Tot. rel. cleft } \\
\text { length }\end{array}$ & $\begin{array}{l}\text { Max cleft } \\
\text { length }\end{array}$ & total cleft length & $\begin{array}{c}\text { Nr. of } \\
\text { Blisters }\end{array}$ \\
\hline Vehicle & $22.4 \%$ & $14 \pm 1 \%$ & $904.21 \mu \mathrm{m}$ & $660.29 \pm 202.81 \mu \mathrm{M}$ & $2-5$ \\
\hline U-73122 & 0 & 0 & $0 \mu \mathrm{m}$ & $0 \pm 0 \mu \mathrm{M}$ & none \\
\hline
\end{tabular}

\section{H\&E}

Vẹicle +lgG Vehicle+PV2

U-73122 +PV2

b)

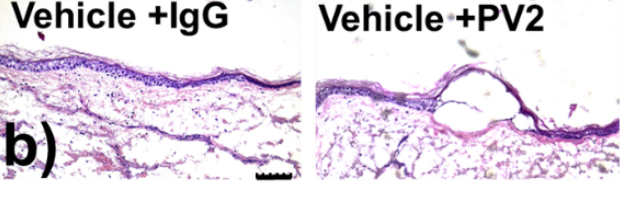

Dsg1
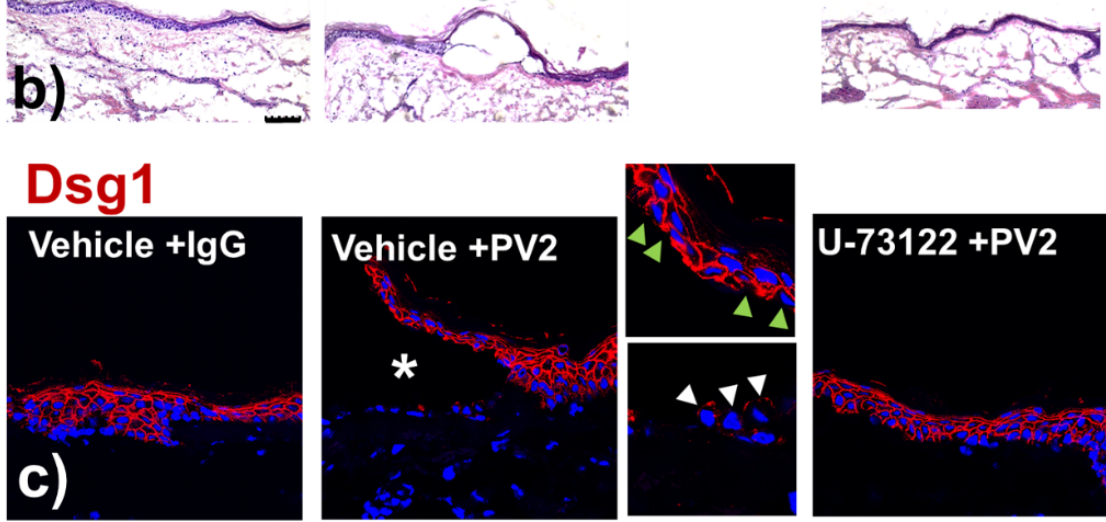

\section{Actin}
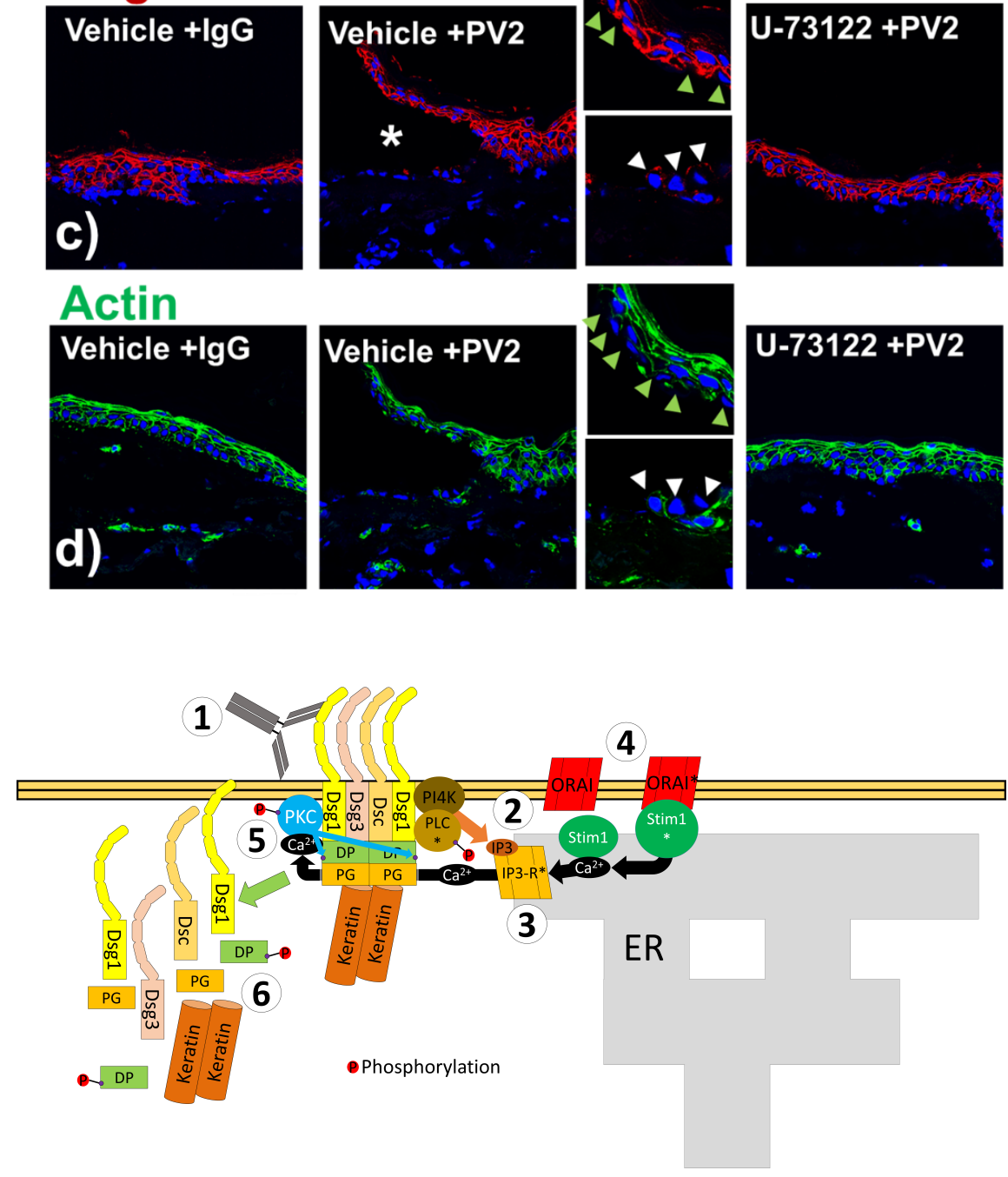